\title{
Exploration of the BaSeL stellar library for 9 F-type stars COROT potential targets ${ }^{\star, \star \star}$
}

\section{Comparisons of fundamental stellar parameter determinations}

\author{
E. Lastennet ${ }^{1,2}$, F. Lignières ${ }^{1,3}$, R. Buser ${ }^{4}$, Th. Lejeune ${ }^{5}$, Th. Lüftinger ${ }^{6}$, \\ F. Cuisinier ${ }^{2}$, and C. van't Veer-Menneret ${ }^{7}$
}

1 Astronomy Unit, Queen Mary and Westfield College, Mile End Road, London E1 4NS, UK

2 Depto. de Astronomia, UFRJ, Ladeira Pedro Antônio 43, 20080-090 Rio de Janeiro RJ, Brazil

${ }^{3}$ Laboratoire d'Astrophysique, CNRS UMR 5572, Obs. Midi-Pyrénées, 57 avenue d'Azereix, 65008 Tarbes Cedex, France

4 Astronomisches Institut der Universität Basel, Venusstr. 7, 4102 Binningen, Switzerland

${ }^{5}$ Observatório Astronómico de Coimbra, Santa Clara, 3040 Coimbra, Portugal

${ }^{6}$ Institut für Astronomie, Universität Wien, Türkenschanzstr. 17, 1180 Wien, Austria

7 Observatoire de Paris, 61 avenue de l'Observatoire, 75014 Paris, France

Received 13 April 2000 / Accepted 19 October 2000

\begin{abstract}
The Basel Stellar Library (BaSeL models) is constituted of the merging of various synthetic stellar spectra libraries, with the purpose of giving the most comprehensive coverage of stellar parameters. It has been corrected for systematic deviations detected in respect to $U B V R I J H K L M$ photometry at solar metallicity, and can then be considered as the state-of-the-art knowledge of the broad band content of stellar spectra. In this paper, we consider a sample of 9 F-type stars with detailed spectroscopic analysis to investigate the Basel Stellar Library in two photometric systems simultaneously, Johnson $(B-V, U-B)$ and Strömgren $(b-y$, $m_{1}$, and $c_{1}$ ). The sample corresponds to potential targets of the central seismology programme of the COROT space experiment, which have been recently observed at OHP. The atmospheric parameters $T_{\text {eff }},[\mathrm{Fe} / \mathrm{H}]$, and $\log g$ obtained from the BaSeL models are compared with spectroscopic determinations as well as with results of other photometric calibrations. For a careful interpretation of the BaSeL solutions, we computed confidence regions around the best $\chi^{2}$-estimates and projected them on $T_{\text {eff- }}[\mathrm{Fe} / \mathrm{H}], T_{\text {eff }}-\log g$, and $\log g$ - $[\mathrm{Fe} / \mathrm{H}]$ diagrams. We first derive the 3 atmospheric parameters from the full photometric information available (Johnson and Strömgren data). The BaSeL library has only been calibrated for broad band UBVRIJHKLM photometry, and it presents therefore intrinsic limitations in respect to other photometric systems, especially with different bandwidth. Thus, using this combination (Johnson and Strömgren), BaSeL temperatures are systematically lower $(\sim 130 \mathrm{~K})$, and the discrepancy for the gravity and the metallicity can be quite large in comparison to the other methods. To disentangle these unexpected discrepancies, we fixed the temperatures at their spectroscopic values and studied the relative influence of each colour index in the $\log g$ - $[\mathrm{Fe} / \mathrm{H}]$ diagrams. We find inconsistent results between Strömgren and Johnson synthetic colours. While the Johnson colours give impressively good log $g$ - $[\mathrm{Fe} / \mathrm{H}]$ solutions, the combination of the $m_{1}$ and $c_{1}$ Strömgren synthetic indices does not provide reliable results. This is not, of course, due to any intrinsic superiority of the $U B V$ over the Strömgren system, but to the properties of the BaSeL models. Finally, in order to simultaneously and accurately determine the stellar parameters $T_{\text {eff }}$, $[\mathrm{Fe} / \mathrm{H}]$ and $\log g$, we suggest to use the combination of the synthetic BaSeL indices $B-V, U-B$ and $b-y$ rather than $B-V, U-B, b-y, m_{1}$ and $c_{1}$.
\end{abstract}

Key words. stars: fundamental parameters - stars: abundances - surveys

\footnotetext{
Send offprint requests to: E. Lastennet

* Based on observations made on the $193 \mathrm{~cm}$ telescope at Observatoire de Haute-Provence, France and data from the ESA Hipparcos satellite.

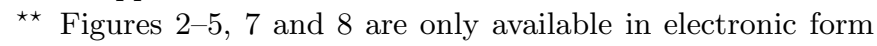
at http://www.edpsciences.org
} 


\section{Introduction}

The Basel Stellar Library (BaSeL) is a library of theoretical spectra corrected to provide synthetic colours consistent with empirical colour-temperature calibrations at all wavelengths from the near-UV to the far-IR (see Cuisinier et al. 1996 for the correction procedure, and Lejeune et al. 1998 and references therein for a complete description). These model spectra cover a large range of fundamental parameters $\left(2000 \leq T_{\text {eff }} \leq 50000 \mathrm{~K},-5 \leq[\mathrm{Fe} / \mathrm{H}] \leq 1\right.$ and $-1.02 \leq \log g \leq 5.5)$ and their photometric calibrations are regularly updated (for instance, the calibrations in the very low metallicity range is being improved by comparisons with globular cluster populations, Westera et al. 1999) and extended to an even larger set of parameters (see Lejeune et al. 1997; Lejeune et al. 1998; Lejeune et al. 2000).

The BaSeL library spectra have been calibrated directly for standard dwarf and giant sequences at solar abundances and using $U B V R I J H K L M$ broad-band photometry, and are hence expected to provide excellent results in these photometric bands. Since they are based on synthetic spectra, they can in principle be used in many other photometric systems taken either individually or simultaneously, and this is another major advantage of these models. For these reasons, they are currently used in an increasing number of astrophysical studies (e.g. Bruzual et al. 1997; Weiss \& Salaris 1999; Kurth et al. 1999).

Nevertheless, little attention has been paid until now to validate the possibilities offered by the BaSeL models in several photometric bands simultaneously. The fact that the library spectra have been calibrated using broadband observations ( $U B V R I J H K L M)$ does not necessarily imply that similarly reliable results can be expected from their application to intermediate- and/or narrow-band photometry data. On the contrary, the extent to which the coarse UBVRIJHKLM calibration provides useful results even at higher resolution requires separate investigation. In this paper, we investigate the BaSeL models using Johnson and Strömgren photometric systems simultaneously. BaSeL Strömgren photometry has been previously tested with success against stars belonging to double-lined eclipsing binaries for which gravities are accurately known (Lastennet et al. 1999a). Here we use a sample of 9 F-type stars for which detailed spectroscopic analysis has been performed. These stars are potential candidates of the COROT (COnvection and ROTation) space experiment main programme (see for instance Catala et al. 1995; Michel et al. 1998 and Baglin et al. 1998 for details), and have been observed at the $193 \mathrm{~cm}$ telescope at Observatoire de Haute-Provence (OHP, France) as part of the target selection process. High signal to noise ratio $(S / N \simeq 150)$ spectra have been obtained using Elodie echelle spectrograph and analysed by comparison with theoretical spectra (Lignières et al. 1999). This set of new spectroscopic data together with both Johnson and Strömgren pho- tometry from the literature provides a unique test of the predicted results of the BaSeL library. In addition, other calibration methods, namely the Templogg programme (based on Napiwotzki et al. 1993; Künzli et al. 1997) and the Marsakov \& Shevelev (1995) catalogue, provide further comparisons.

The paper is organized as follows: Sect. 2 deals with the description of our working sample of stars and the extinction issue, Sect. 3 presents the various methods used to derive estimates of $T_{\text {eff }},[\mathrm{Fe} / \mathrm{H}]$, and $\log g$, and Sect. 4 is devoted to the presentation and the discussion of the results, with particular emphasis put on the intrinsic properties of the BaSeL library spectra in Sect. 5. Finally, Sect. 6 draws our general conclusions.

\section{The sample: relevant data and reddening}

\subsection{Basic data for the 9 stars}

The photometric data in the Johnson $(B-V$ and $U-B)$ and Strömgren $\left(b-y, m_{1}, c_{1}\right)$ systems of our working sample of F-type stars are presented in Table 1, along with cross-identifications, Hipparcos parallaxes $(\pi)$ and rotational velocities $(v \sin i$ is derived from the application of the Least-Squares Deconvolution method of Donati et al. 1997 on the OHP spectra).

\subsection{Reddening}

Estimates of the extinction are required for photometric calibration methods and in particular for the BaSeL and Templogg methods used in this paper. Parallaxes listed in Table 1 from the Hipparcos catalogue (ESA, 1997, see also Perryman et al. 1997) show that all the stars are in the close solar neighborhood $(\leq 45 \mathrm{pc})$. While reddening is generally expected to be close to zero inside this very local sphere (e.g. Welsh et al. 1991), we checked this by applying the extinction model of Vergely (1998) to our stars. This model has been constructed from a sample of 4000 stars (in a sphere of $250 \mathrm{pc}$ ) with Strömgren photometry and provides extinction as a function of position and distance ${ }^{1}$. The $\sigma_{\pi} / \pi$ values $(\leq 3.3 \%)$ reported for the present stars in the Hipparcos Catalogue ensure that our extinction estimates cannot be seriously affected by distances uncertainties. Results are shown in Table 1 (Col. $E(b-y)$ ) and confirm that the reddening is very close to zero for all stars. Such small values have no influence on the inferred photometric results presented in this paper, and will thus be neglected. Therefore, all the results obtained in the remainder of this paper will be given without reddening.

\footnotetext{
${ }^{1}$ However, it is also worth noticing that this model neglect small clumps and circumstellar extinction, which means that if there is a small clump with a high density on the line of sight, the model underestimates the extinction.
} 
Table 1. Cross-identifications (HD and HIP numbers), Hipparcos parallaxes (Perryman et al. 1997), extinction $(E(b-y)$ from Vergely 1998), rotational velocities $v \sin i\left(\mathrm{~km} \mathrm{~s}^{-1}\right)$, and photometric data used in the BaSeL and Templogg determinations for the 9 target stars

\begin{tabular}{rrrrlrrrrrr}
\hline $\mathrm{ID}^{\dagger}$ & $\mathrm{HD}$ & $\mathrm{HIP}$ & $\pi$ (mas) & $E(b-y)$ & $v \sin i$ & $B-V$ & $U-B$ & $b-y$ & $m_{1}$ & $c_{1}$ \\
\hline 1 & 43587 & 29860 & $51.76 \pm 0.78$ & 0.001 & 2 & $0.61 \pm 0.01$ & $0.10 \pm 0.01$ & $0.384 \pm 0.01$ & $0.187 \pm 0.03$ & $0.349 \pm 0.03$ \\
2 & 43318 & 29716 & $28.02 \pm 0.76$ & 0.002 & 5 & $0.49 \pm 0.01$ & $0.00 \pm 0.01$ & $0.322 \pm 0.03$ & $0.154 \pm 0.03$ & $0.446 \pm 0.03$ \\
3 & 45067 & 30545 & $30.22 \pm 0.92$ & 0.001 & 6 & $0.56 \pm 0.01$ & $0.07 \pm 0.01$ & $0.361 \pm 0.02$ & $0.168 \pm 0.03$ & $0.396 \pm 0.03$ \\
4 & 49933 & 32851 & $33.45 \pm 0.84$ & 0.001 & 10 & $0.39 \pm 0.01$ & $-0.09 \pm 0.02$ & $0.270 \pm 0.02$ & $0.127 \pm 0.04$ & $0.460 \pm 0.03$ \\
5 & 49434 & 32617 & $24.95 \pm 0.75$ & 0.002 & 79 & $0.295 \pm 0.015$ & $0.05 \pm 0.03$ & $0.178 \pm 0.01$ & $0.178 \pm 0.02$ & $0.717 \pm 0.03$ \\
6 & 46304 & 31167 & $23.13 \pm 0.76$ & 0.002 & 200 & $0.25 \pm 0.01$ & $0.06 \pm 0.01$ & $0.158 \pm 0.01$ & $0.175 \pm 0.02$ & $0.816 \pm 0.04$ \\
7 & 162917 & 87558 & $31.87 \pm 0.77$ & 0.004 & 25 & $0.42 \pm 0.01$ & $-0.03 \pm 0.02$ & $0.280 \pm 0.02$ & $0.166 \pm 0.06$ & $0.458 \pm 0.01$ \\
8 & 171834 & 91237 & $31.53 \pm 0.75$ & 0.004 & 64 & $0.37 \pm 0.01$ & $-0.04 \pm 0.01$ & $0.254 \pm 0.04$ & $0.145 \pm 0.01$ & $0.560 \pm 0.04$ \\
9 & 164259 & 88175 & $43.11 \pm 0.75$ & 0.003 & 76 & $0.38 \pm 0.01$ & $-0.01 \pm 0.01$ & $0.253 \pm 0.01$ & $0.153 \pm 0.01$ & $0.560 \pm 0.04$ \\
\hline
\end{tabular}

$\dagger$ Arbitrary running number.

\section{Description of the different methods}

In this section, we present the various methods used to derive $T_{\text {eff }},[\mathrm{Fe} / \mathrm{H}]$, and $\log g$. Apart from the BaSeL models, a detailed spectroscopic analysis and two photometric calibrations have been considered.

\subsection{Photometric analysis}

\subsubsection{The BaSeL models}

For the present work, more than 50700 models have been computed by interpolation by two of us (Lastennet and Lejeune), each model giving synthetic photometry in the Johnson and Strömgren systems for a set of $\left(T_{\text {eff }},[\mathrm{Fe} / \mathrm{H}]\right.$, $\log g)$. In order to fit the observed colours of the target stars, we have computed a fine grid in the $\left(T_{\text {eff }},[\mathrm{Fe} / \mathrm{H}]\right.$, $\log g$ ) parameter space. The grid explored is defined in this way: $5000 \leq T_{\text {eff }} \leq 8000 \mathrm{~K}$ in $20 \mathrm{~K}$ steps, $-1 \leq$ $[\mathrm{Fe} / \mathrm{H}] \leq 0.5$ in 0.1 steps, and $3 \leq \log g \leq 5$ in 0.1 steps. These parameter ranges are reasonable matches to the expected properties of $\mathrm{F}$ dwarfs, for which the BaSeL library includes the Kurucz atmospheric models (Mixing Length Theory of convection with $l / H_{\mathrm{P}}=1.25$, and with the overshooting parameter equal to 1 ).

In order to derive simultaneously the effective temperature $\left(T_{\text {eff }}\right)$, the metallicity $([\mathrm{Fe} / \mathrm{H}])$, and the surface gravity $(\log g)$ of each star, we minimize the $\chi^{2}$-functional defined as:

$\chi^{2}\left(T_{\mathrm{eff}},[\mathrm{Fe} / \mathrm{H}], \log g\right)=\sum_{i=1}^{5}\left[\left(\frac{\operatorname{col}(i)_{\bmod }-\operatorname{col}(i)}{\sigma(\operatorname{col}(i))}\right)^{2}\right]$

where $\operatorname{col}(i)$ and $\sigma(\operatorname{col}(i))$ are the observed values $(B-V$, $U-B, b-y, m_{1}$, and $\left.c_{1}\right)$ and their error bars, as reported in Table 1 , and $\operatorname{col}(i)_{\bmod }$ are obtained from the synthetic computations of the BaSeL models. A similar method has already been developed and used by Lastennet et al. (1996, 1999b) for CMD diagrams.

With 5 observational data $\left(B-V, U-B, b-y, m_{1}\right.$ and $c_{1}$ for each star) and 3 free parameters $\left(T_{\text {eff }}, \log g\right.$ and $[\mathrm{Fe} / \mathrm{H}])$, we expect to find a $\chi^{2}$-distribution with 2 degrees of freedom. Since the $\chi^{2}$-value is an estimation of the quality of the fit, with 2 degrees of freedom (DOF), a $\chi^{2}-$ value smaller than 6 is a good fit, because it means that the probability $P$ that this $\chi^{2} \mathrm{DOF}=2$ is smaller than 6 is about $95 \%$, i.e. $P\left(\chi^{2}\right.$ DOF=2 $)<6 \simeq 95 \%$. This criterion will change according to the DOF, but basically, small values are signatures of good fits. In the remainder of this paper, the $\chi^{2}$-values generally satisfy this criterion being most of the time much better, hence by default they will not be discussed when they are good. A $\chi^{2}$-grid is formed in the $\left(T_{\text {eff }}, \log g,[\mathrm{Fe} / \mathrm{H}]\right)$ parameter space. Once the central minimum value $\chi_{\min }^{2}$ is found, we compute the surfaces corresponding to $1 \sigma, 2 \sigma$, and $3 \sigma$ confidence levels. For clarity, the intersection of these confidence surfaces with the appropriate plane will be displayed (e.g. $T_{\text {eff }}-[\mathrm{Fe} / \mathrm{H}]$ in Fig. 1, $T_{\text {eff- }} \log g$ in Fig. 2).

\subsubsection{The "Templogg" method}

We have also run the "Templogg" program which is designed to determine effective temperature and log g from either Strömgren or Geneva photometry. For Strömgren photometry it uses a Fortran program written by E. Fresno which relies upon the grids of Moon \& Dworetsky (1985) in the $T_{\text {eff }}-\log g$ parameter space relevant to this paper, with the improvements by Napiwotzki et al. (1993). For Geneva photometry it uses a Fortran program written by M. Kunzli (see North et al. 1994). The program chooses among eight different regions in the HR diagram for selecting the best calibration within the Strömgren system and three different regions for the Geneva system. The results from this method are gathered in Table 2 .

\subsubsection{The catalogue of Marsakov \& Shevelev (1995)}

Marsakov \& Shevelev (1995) (hereafter [MS95]) have computed effective temperatures and surface gravities using Moon's (1985) method, which is also based on the interpolation of the grids presented in Moon \& Dworetsky (1985). 
According to Moon (1985), the standard deviations of the derived parameters are $T_{\text {eff }}= \pm 100 \mathrm{~K}$ and $\log g= \pm 0.06$. The metallicities of Marsakov \& Shevelev (1995) are obtained with the equation of Carlberg et al. (1985):

$$
[\mathrm{Fe} / \mathrm{H}]=0.16-0.66 \times \Delta \beta-[12.3-38 \times \Delta \beta] \times \delta m_{1},
$$

where $\Delta \beta=2.72-\beta$, and the colour excess $\delta m_{1}$ and the Strömgren $\beta$ index are determined from $(b-y)$. All the [MS95] results relevant for our sample are given in Table 2.

\subsection{Spectroscopic analysis}

The fundamental stellar parameters have been derived from a detailed analysis of spectra with high signal to noise ratio $(S / N \simeq 150)$ obtained at $\mathrm{OHP}$ with the Elodie echelle spectrograph (spectra ranging from $3906 \AA$ to $6811 \AA$ at a resolution of $\lambda / \Delta \lambda=42000)$. After reduction, the observed spectra were compared with theoretical ones constructed from a combination of Kurucz atmospheric models (ATLAS9 - Mixing Length Theory of convection with $l / H_{\mathrm{P}}=0.5$ and without overshooting. This choice of parameters different than those used by Kurucz is fully justified in van't Veer-Menneret \& Mégessier (1996)), the VALD-2 atomic database (Kupka et al. 1999), and the SYNTH radiative transfer codes (Piskunov 1992) and BALMER9 (Kurucz 1993). In addition, the Least-Squares Deconvolution method (Donati et al. 1997) provided accurate determination of the projected rotational velocities listed in Table 1. Details about these determinations are given in the two next subsections and the results are summarized in Tables 1 and 2. Note that the level of accuracy of these results, initially designed to select COROT targets, is sufficient for the purpose of the present study.

\subsubsection{Determination of $T_{\text {eff }}$ from the $\mathrm{H} \alpha$ line}

The effective temperature can be determined by taking advantage of the sensitivity of $\mathrm{H} \alpha$ line wings. Most importantly, detailed studies (e.g. van't Veer-Menneret \& Mégessier 1996), have shown that the $\mathrm{H} \alpha$ line is independent of the surface gravity (for non-supergiant stars) for effective temperatures ranging from $5000 \mathrm{~K}$ to $\sim 8500 \mathrm{~K}$, and depends only slightly on the metallicity. $T_{\text {eff }}$ is therefore obtained for each star of the sample by fitting the observed $\mathrm{H} \alpha$ line with synthetic spectra computed from a grid of solar metallicity atmospheric models separated by $250 \mathrm{~K}$.

\subsubsection{Determination of the surface gravity and metallicity}

Within the temperature range that we found, we noticed that Fe I absorption lines depend only on temperature and metallicity, being practically independent of the surface gravity, while Fe II lines are sensitive to the temperature, metallicity and gravity. Consequently, the temperature being known from the $\mathrm{H} \alpha$ line, the metallicity along with the microturbulence velocity are determined first by fitting a set of weak and strong Fe I lines. Then, the gravity is obtained by fitting Fe II lines. The spectral region near $6130 \AA$ proved to be suitable for this analysis. However, the line broadening induced by rotation tends to mix neighbouring lines and prevent the analysis of individual Fe lines for high values of $v \sin i$ (HD 49434, HD 46304, HD 171834 and HD 164259).

\subsection{Other determinations in the literature}

To be as complete as possible, we looked for other determinations available in the literature and the SIMBAD database. One of the most comprehensive sources for our purpose is the fifth Edition of the catalogue of Cayrel de Strobel et al. (1997), which includes $[\mathrm{Fe} / \mathrm{H}]$ determinations and atmospheric parameters $\left(T_{\text {eff }}, \log g\right.$ ) obtained from high-resolution spectroscopic observations and detailed analyses, most of them carried out with the help of model atmospheres. However, the stars of our sample are not included in this catalogue. Since the catalogue comprises the literature (700 bibliographical references) up to December 1995, we only looked for more recent references. To the best of our knowledge, the catalogue of metallicities of Zakhozhaj \& Shaparenko (1996) (hereafter [ZS96]) is the only one which contains useful information for our purpose. These metallicities are obtained from photometric $U B V$ data and are available for two stars of our sample: HD 43587 and HD 164259 (see Table 2).

\section{Multi-colour exploration of the BaSeL models: Comparisons and discussion}

In this section, we will discuss the results obtained from the exploration of the BaSeL model properties in the Johnson and Strömgren photometric systems (the best solutions from the BaSeL models and from the other methods are summarized in Table 2). First we discuss the results in the $T_{\text {eff }}, \log g,[\mathrm{Fe} / \mathrm{H}]$ parameter space using all the photometric indices. Discrepancies between synthetic and spectroscopic results led us to consider solutions derived from Johnson and Strömgren photometry separately, by fixing (in the second part of this discussion) $T_{\text {eff }}$ at its spectroscopic values and investigating the BaSeL properties in $\log g$ - $[\mathrm{Fe} / \mathrm{H}]$ diagrams. Finally, we present the most reliable combination of synthetic photometry indices to get $T_{\text {eff }}, \log g$ and $[\mathrm{Fe} / \mathrm{H}]$ simultaneously.

\subsection{Simultaneous $T_{\text {eff }}, \log g,[\mathrm{Fe} / \mathrm{H}]$ determinations using all the photometric indices}

The results obtained with all the methods described in Sect. 3 are shown in $T_{\text {eff }}-[\mathrm{Fe} / \mathrm{H}]$ diagrams (Fig. 1) and $T_{\text {eff }}-\log g$ diagrams (Fig. 2) for the 9 stars of our sample. The solutions from the BaSeL models (i.e. the best $\chi^{2}$ solution plus the 1-, 2- and 3- $\sigma$ confidence level contours) 
Table 2. Comparison of fundamental stellar parameters determinations. The Templogg method uses Strömgren and Geneva photometric data, Marsakov \& Shevelev (1995) ([MS95]) used Strömgren data, and the BaSeL method uses Johnson and Strömgren data. Results from our spectroscopic analysis (Spectro.), and Zakhozhaj \& Shaparenko (1996) [ZS96] are also given

\begin{tabular}{|c|c|c|c|c|c|c|c|c|c|c|}
\hline $\begin{array}{l}\mathrm{ID}^{\dagger} \\
\mathrm{HD}\end{array}$ & & $\begin{array}{c}1 \\
43587\end{array}$ & $\begin{array}{c}2 \\
43318\end{array}$ & $\begin{array}{c}3 \\
45067\end{array}$ & $\begin{array}{c}4 \\
49933\end{array}$ & $\begin{array}{c}5 \\
49434\end{array}$ & $\begin{array}{c}6 \\
46304\end{array}$ & $\begin{array}{c}7 \\
162917\end{array}$ & $\begin{array}{c}8 \\
171834\end{array}$ & $\begin{array}{c}9 \\
164259\end{array}$ \\
\hline & Method & & & & & & & & & \\
\hline$T_{\text {eff }}$ & $\begin{array}{l}\text { Templogg } \\
\text { [MS95] } \\
\text { BaSeL }^{a} \\
\text { BaSeL }^{c} \\
\text { Spectro. }\end{array}$ & $\begin{array}{l}6009 \\
5952 \\
5740 \\
5720 \\
6000\end{array}$ & $\begin{array}{l}6420 \\
6280 \\
6040 \\
6320 \\
6250\end{array}$ & $\begin{array}{l}5982 \\
6066 \\
6000 \\
5940 \\
6000\end{array}$ & $\begin{array}{l}6535 \\
6625 \\
6420 \\
6600 \\
6500\end{array}$ & $\begin{array}{l}7321 \\
7120 \\
7240 \\
7250\end{array}$ & $\begin{array}{l}7379 \\
7200 \\
7240 \\
7250\end{array}$ & $\begin{array}{l}6587 \\
6629 \\
6400 \\
6660 \\
6500\end{array}$ & $\begin{array}{l}6714 \\
6739 \\
6580 \\
6700 \\
6750\end{array}$ & $\begin{array}{l}6789 \\
6730 \\
6580 \\
6820 \\
6750\end{array}$ \\
\hline $\log g$ & $\begin{array}{l}\text { Templogg } \\
\text { [MS95] } \text { BaSeL }^{a} \\
\text { BaSeL }^{b} \\
\text { BaSeL }^{c} \\
\text { Spectro. }^{\ddagger}\end{array}$ & $\begin{array}{c}4.32 \\
4.11 \\
3.4 \\
\text { bad fit } \\
4.3 \\
4.5\end{array}$ & $\begin{array}{l}4.20 \\
4.05 \\
3.1 \\
4.5 \\
4.5 \\
4.0\end{array}$ & $\begin{array}{c}4.16 \\
4.02 \\
3.9 \\
3.8 \\
3.8 \\
4.0\end{array}$ & $\begin{array}{l}4.25 \\
4.46 \\
3.5 \\
3.9 \\
4.3 \\
4.0\end{array}$ & $\begin{array}{l}3.5 \\
4.0 \\
4.0\end{array}$ & $\begin{array}{l}3.93 \\
\\
3.3 \\
3.4 \\
3.4\end{array}$ & $\begin{array}{l}4.32 \\
4.49 \\
3.6 \\
4.0 \\
4.5 \\
4.0\end{array}$ & $\begin{array}{c}4.02 \\
4.10 \\
3.4 \\
4.1 \\
3.9\end{array}$ & $\begin{array}{l}4.11 \\
4.10 \\
3.3 \\
4.0 \\
4.2\end{array}$ \\
\hline$[\mathrm{Fe} / \mathrm{H}]$ & $\begin{array}{l}\text { Templogg } \\
\text { [MS95] } \\
\text { BaSeL }^{a} \\
\text { BaSeL }^{b} \\
\text { BaSeL }^{c} \\
\text { Spectro. }^{\ddagger} \\
\text { [ZS96] }^{\text {a }}\end{array}$ & $\begin{array}{c}-0.13 \\
-0.15 \\
-0.2 \\
\text { bad fit } \\
-0.2 \\
-0.1 \\
+0.03\end{array}$ & $\begin{array}{l}-0.18 \\
-0.18 \\
-0.5 \\
-0.1 \\
+0.0 \\
-0.3\end{array}$ & $\begin{array}{l}-0.15 \\
-0.17 \\
+0.0 \\
+0.0 \\
-0.1 \\
-0.1\end{array}$ & $\begin{array}{l}-0.48 \\
-0.35 \\
-1.0 \\
-0.8 \\
-0.6 \\
-0.5\end{array}$ & $\begin{array}{l}-0.03 \\
-0.5 \\
-0.1 \\
-0.1\end{array}$ & $\begin{array}{l}-0.09 \\
-0.9 \\
-0.8 \\
-0.8\end{array}$ & $\begin{array}{l}+0.03 \\
+0.08 \\
-0.5 \\
-0.3 \\
+0.0 \\
-0.2\end{array}$ & $\begin{array}{l}-0.20 \\
-0.15 \\
-0.8 \\
-0.4 \\
-0.5\end{array}$ & $\begin{array}{l}-0.11 \\
-0.05 \\
-0.6 \\
-0.2 \\
+0.0 \\
-0.03\end{array}$ \\
\hline
\end{tabular}

${ }^{\dagger}$ Running number as in Table 1.

${ }^{a}$ From $U B V$ Johnson $(B-V$ and $U-B)$ and Strömgren $\left(b-y, m_{1}\right.$ and $\left.c_{1}\right)$ data (cf. Fig. 1 or Fig. 2).

${ }^{b}$ From $U B V$ Johnson data (Fig. 3).

${ }^{c}$ From the combination $B-V, U-B$ and $b-y$ (Figs. 7 and 8 ).

${ }^{\ddagger}$ Estimated error: $\Delta T_{\text {eff }} \simeq \pm 250 \mathrm{~K}, \Delta \log g \simeq \pm 0.5, \Delta[\mathrm{Fe} / \mathrm{H}] \simeq \pm 0.2$.

are obtained in order to fit simultaneously the 5 available observed photometric values of Table 1: $(B-V),(U-B)$, $(b-y), m_{1}$, and $c_{1}$. For each star, the contour solutions are displayed in a $\log g=$ constant plane (Fig. 1) or an $[\mathrm{Fe} / \mathrm{H}]=$ constant plane (Fig. 2), corresponding to the best simultaneous $\left(T_{\text {eff }},[\mathrm{Fe} / \mathrm{H}], \log g\right)$ solutions derived from the BaSeL models. When available, the results from the spectroscopic analysis (diamond with error bars) as well as from the "Templogg" programme (square), and Marsakov \& Shevelev (1995) (triangle) are projected in these diagrams for comparison.

As far as the effective temperature is concerned, the overall agreement is good between all the methods. However, with comparison to spectroscopy, the BaSeL models give systematically lower values: $\left\langle\Delta T_{\text {eff }}>=\right.$ $<T_{\text {eff }}$ (spectro) $-T_{\text {eff }}(\mathrm{BaSeL})>=130 \mathrm{~K}$ on average (which is comparable to the result of Cuisinier et al. 1994). On the other hand, the Templogg method gives systematically larger values (by $\sim 60 \mathrm{~K}$ on average). Results given by either the [MS95] catalogue or the Templogg method are essentially identical. There is no such overall agreement for the surface gravity and the metallicity, and this disagreement is neither correlated with increasing temperature nor with increasing $v \sin i$. For instance in Fig. 2, while the systematic disagreement in gravity appears for stars with the hottest spectroscopic temperatures and high rotation speeds (HD 49434, HD 46304, HD 171834 and HD 164259), the disagreement is even worse for the cooler and slowly rotating star HD 43318. Thus, except in one case (HD 45067) where the match is perfect for both $\log$ and $[\mathrm{Fe} / \mathrm{H}]$, BaSeL-derived metallicities and gravities are systematically lower than the values obtained from the other methods. In particular, the best- $\chi^{2}$ values of $\log g$ inferred from the synthetic BaSeL colours range between 3.1 and 3.9, indicating possible evolved/sub-giant stars, which is ruled out by spectroscopic determinations (listed in Table 2). Even if inspection of the confidence contours might temper this conclusion by pointing out that the BaSeL results are sometimes compatible to $3-\sigma$, the systematic behaviour remains, at least for the small sample studied, and requires further analysis.

\subsection{Simultaneous $[\mathrm{Fe} / \mathrm{H}]-\log g$ determinations using various combinations of photometric indices}

Since it appears that the BaSeL models have difficulties reproducing simultaneously the 3 parameters with all the photometric information (Strömgren plus Johnson), 

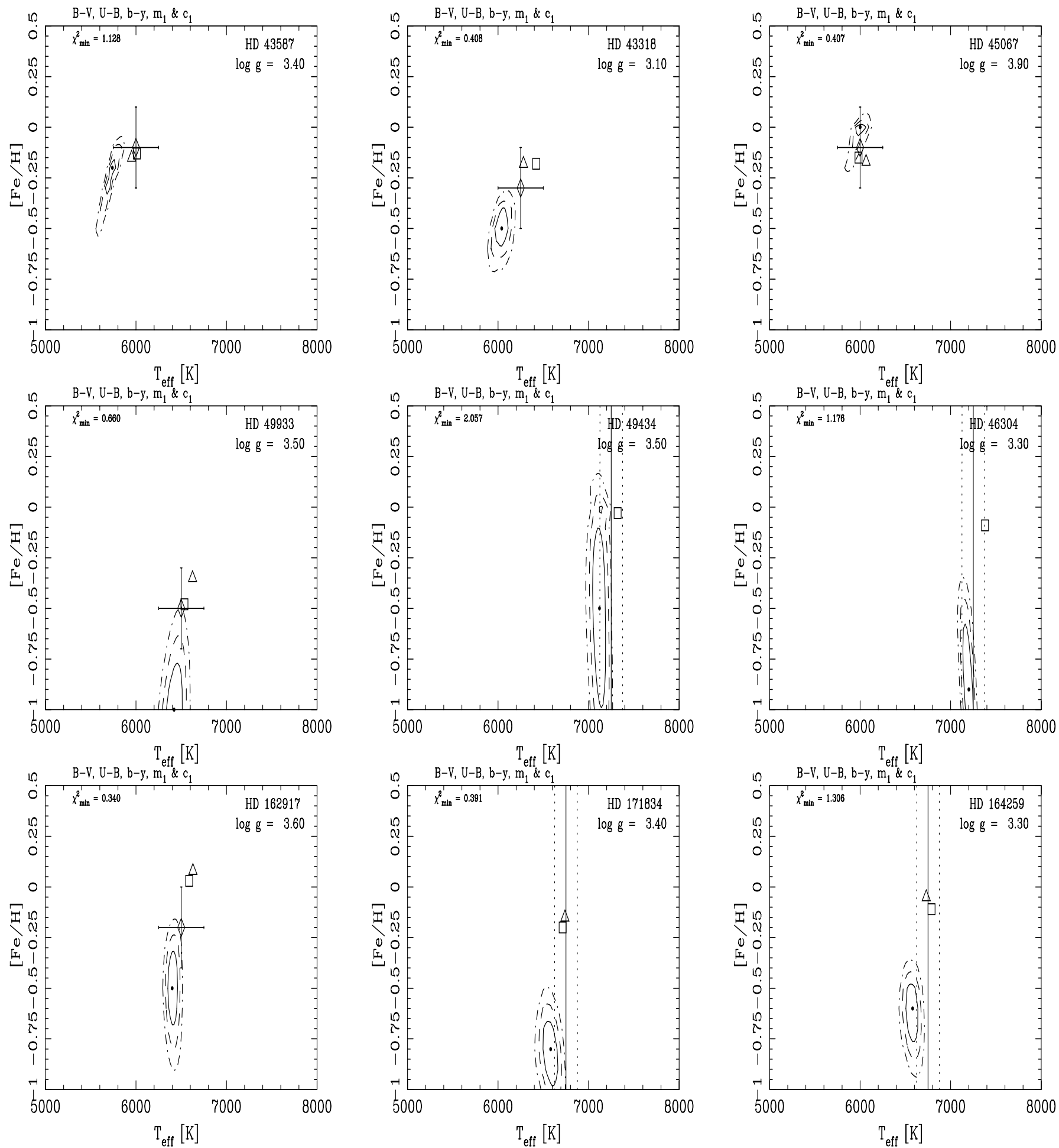

Fig. 1. Simultaneous $\left(T_{\text {eff }},[\mathrm{Fe} / \mathrm{H}]\right)$ results for the 9 potential targets of the COROT central seismology programme. The solutions from the BaSeL models (1-, 2- and 3- $\sigma$ confidence level contours) are obtained in order to fit simultaneously the 5 following observed photometric values: $(B-V),(U-B),(b-y), m_{1}$ and $c_{1}$. For each star, the contour solutions are displayed in a $\log g=$ constant plane, corresponding to the best simultaneous $\left(T_{\text {eff }},[\mathrm{Fe} / \mathrm{H}], \log g\right)$ solutions derived from the BaSeL models (the grid explored is: $5000 \leq T_{\text {eff }} \leq 8000 \mathrm{~K}$ in $20 \mathrm{~K}$ steps, $-1 \leq[\mathrm{Fe} / \mathrm{H}] \leq 0.5$ in 0.1 steps, and $3 \leq \log g \leq 5$ in 0.1 steps). An estimation of the quality of the best fit $\left(\chi^{2}\right.$-value) is also quoted in each panel. The results projected in the $T_{\text {eff }}-[\mathrm{Fe} / \mathrm{H}]$ planes from the spectroscopic analysis (diamond with error bars, or solid plus two dotted lines) as well as from the "Templogg" programme (square), and Marsakov \& Shevelev (1995) (triangle) are also shown for comparison 
we checked if this remains true using either Johnson or Strömgren photometry separately, in order to find out which photometric strategy would improve the situation. As only two Johnson indices are available in the data (Table 1), namely $B-V$ and $U-B$, the three fundamental parameters cannot be derived simultaneously using Johnson photometry alone. Therefore, we choose to fix the effective temperature derived from spectroscopy, and results for Johnson (Fig. 3) and Strömgren (Fig. 4) are compared in $\log g-[\mathrm{Fe} / \mathrm{H}]$ diagrams. Note that the $m_{1}$ and $c_{1}$ indices are optimized linear combinations of colour indices designed for the purposes of measuring metallicity and surface gravity, respectively, whereas $U B V$ was not designed at all on such physical considerations. Therefore, the $m_{1}$ and $c_{1}$ indices should a priori give the best determinations of $[\mathrm{Fe} / \mathrm{H}]$ and $\log g$.

The effective temperature being fixed to its spectroscopic value, the metallicity and the surface gravity of each star are determined simultaneously by minimizing the following $\chi^{2}$-functional:

$\chi^{2}([\mathrm{Fe} / \mathrm{H}], \log g)=\sum_{i=1}^{2}\left[\left(\frac{\operatorname{col}(i)_{\bmod }-\operatorname{col}(i)}{\sigma(\operatorname{col}(i))}\right)^{2}\right]$

where $\operatorname{col}(i)$ and $\sigma(\operatorname{col}(i))$ are the observed values and their error bars respectively, and $\operatorname{col}(i)_{\bmod }$ are obtained from the synthetic computations of the BaSeL models.

Figures 3 and 4 display the $\log g$ - $[\mathrm{Fe} / \mathrm{H}]$ solutions for Johnson and Strömgren, respectively. The impressively good results of Fig. 3 show that for the spectroscopic temperatures, the BaSeL library spectra provide $\log g$ values which deviate from the spectroscopic values neither by a significant amount, nor in a systematic manner.

A similar, only slightly weaker agreement holds for the metallicities: they come out within less than $\sim 0.2$ dex from the spectroscopic results, which is excellent in view of the intrinsic accuracy $(\sim 0.2 \mathrm{dex})$ that can be obtained from empirical $U B V$ data and calibrations (e.g. Buser \& Kurucz 1992).

Direct comparison of Fig. 3 with Fig. 4 - which uses the information on all the Strömgren indices - clearly shows that the results from Johnson and Strömgren are not compatible. In most cases, even the 1- $\sigma$ contours are partly outside the $\log g$-[Fe/H] diagrams in Fig. 4, suggesting that the best solutions inferred from the 3 Strömgren synthetic indices are perhaps even further away from the expected values. We performed various tests to identify which synthetic Strömgren index (indices) is (are) responsible for the disagreement with the Johnson solutions. We add separately each Strömgren index to the $U B V$ data, in order to highlight the influence of each of them on the contours derived from the Johnson data. The indices $m_{1}$ and $c_{1}$ are clearly found to modify the Johnson contours, in the sense of degrading the results. On the other hand, $b-y$ does not modify the $U B V$ confidence contours. This last point is illustrated by comparing Fig. $3(B-V, U-B)$ with Fig. $5(B-V, U-B$ and $b-y)$. We observe that neither the contours nor the $\chi_{\min }$ values are modified by
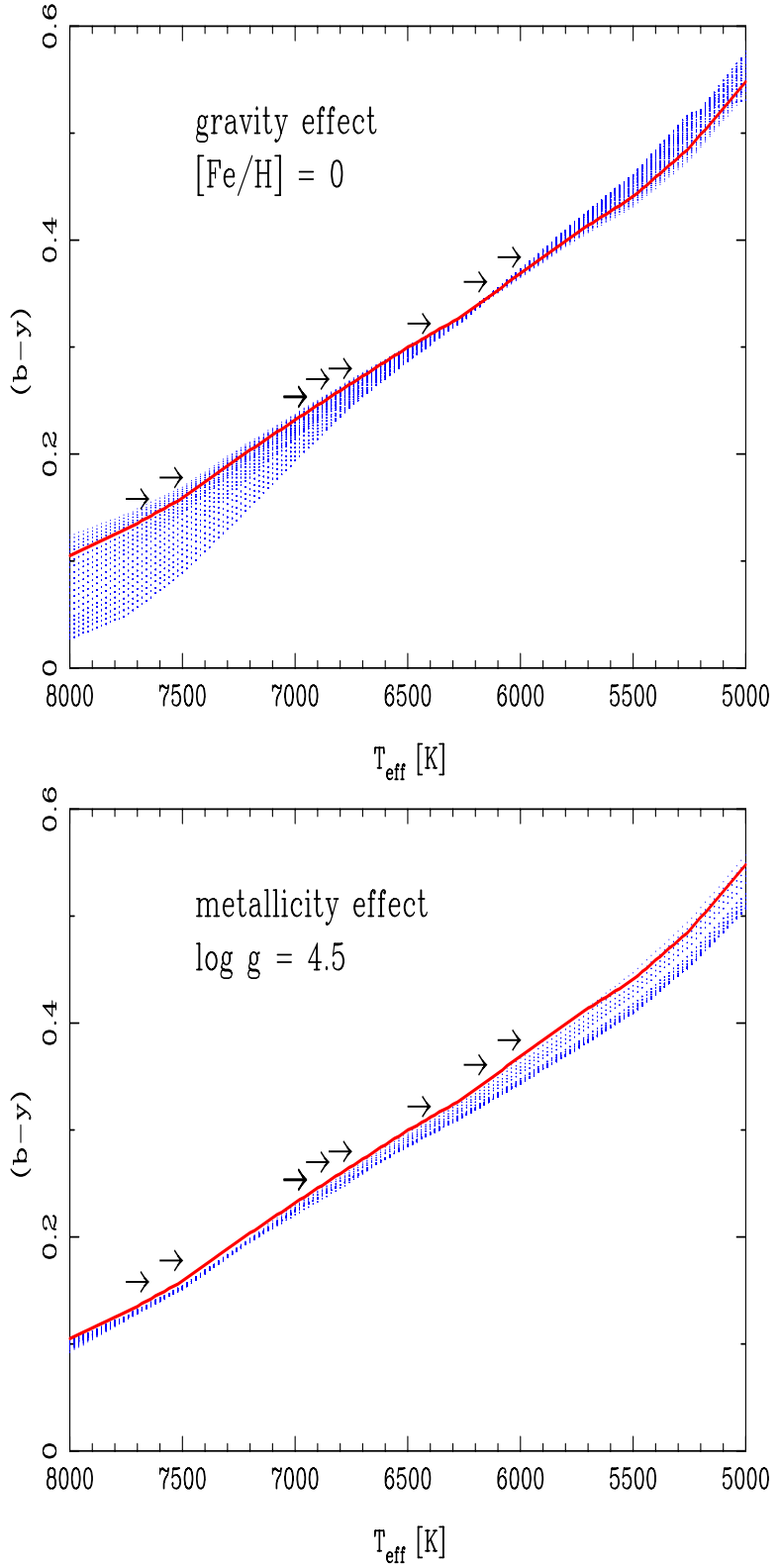

Fig. 6. $T_{\text {eff- }}(b-y)$ BaSeL relationship for the effective temperature range $(5000-8000 \mathrm{~K})$ relevant for this work. On the top panel the effect of gravity (dashed area) is shown for $[\mathrm{Fe} / \mathrm{H}]$ $=0$ and $\log g$ values between 3 and 5 , and on the lower panel the effect of metallicity (dashed area) is shown for log $g=4.5$ and $[\mathrm{Fe} / \mathrm{H}]$ between -1 . and 0.5 . For comparison a $[\mathrm{Fe} / \mathrm{H}]=0$ and $\log g=4.5$ model (bold solid line), and the $(b-y)$ values (arrows) of the sample stars are shown on both panels

the addition of the $b-y$ index to the $B-V$ and $U-B$ indices.

The reason of this remarkable property is twofold: first, the $b-y$ index depends only slightly on $\log g$ and $[\mathrm{Fe} / \mathrm{H}]$ in the parameter range considered; and second, the BaSeL $T_{\text {eff }}$ associated with $b-y$ is fully compatible with the spectroscopic $T_{\text {eff }}$ for the 9 stars. The first point is illustrated in Fig. 6 in the effective temperature range 5000-8000 K: the upper panel shows the effect of gravity ( $\log g$ between 3 and 5) for constant metallicity $([\mathrm{Fe} / \mathrm{H}]=0)$, and the lower 
Table 3. Comparison of $T_{\text {eff }}$ (spectro) vs. $T_{\text {eff }}(\mathrm{BaSeL})$ using $b-y$ only. The two values from the BaSeL models correspond to the maximum width due to the effect of surface gravity (at $[\mathrm{Fe} / \mathrm{H}]=0$ ) and the effect of metallicity (at $\log g=4.5$ ) as shown in Fig. 6

\begin{tabular}{rrccc}
\hline ID $^{\dagger}$ & HD & $T_{\text {eff }}^{\ddagger}$ & \multicolumn{2}{c}{$T_{\text {eff }}($ BaSeL) } \\
& & (spectro) & $3 \leq \log g \leq 5$ & $-1 \leq[\mathrm{Fe} / \mathrm{H}] \leq 0.5$ \\
\hline 1 & 43587 & 6000 & $5860-5940$ & $5680-5910$ \\
2 & 43318 & 6250 & $6260-6315$ & $6160-6320$ \\
3 & 45067 & 6000 & $6020-6070$ & $5850-6060$ \\
4 & 49933 & 6500 & $6590-6750$ & $6610-6730$ \\
5 & 49434 & 7250 & $7055-7440$ & $7305-7380$ \\
6 & 46304 & 7250 & $7150-7610$ & $7440-7510$ \\
7 & 162917 & 6500 & $6525-6665$ & $6525-6650$ \\
8 & 171834 & 6750 & $6685-6875$ & $6730-6850$ \\
9 & 164259 & 6750 & $6685-6875$ & $6730-6850$ \\
\hline
\end{tabular}

$\dagger$ Running number as in Table 1 .

‡ Estimated error: $\Delta T_{\text {eff }} \simeq \pm 250 \mathrm{~K}$.

one the effect of metallicity $([\mathrm{Fe} / \mathrm{H}]$ between -1 and 0.5$)$ for constant gravity $(\log g=4.5)$. It appears clearly from these two plots that the $T_{\text {eff }}=f(b-y)$ relationship is little influenced by surface gravity between $\sim 5800$ and $6700 \mathrm{~K}$, and by metallicity above $6500 \mathrm{~K}$. The second point is proved in Table 3 , where $T_{\text {eff }}$ derived from the BaSeL calibrations shown in Fig. 6 are in agreement with the spectroscopic determinations. This underlines the reliability of temperature determinations based on the synthetic $b-y$ index derived from the BaSeL models.

In conclusion, we find that synthetic Strömgren $m_{1}$ and $c_{1}$ account for the difference between the Johnson and Strömgren solutions. We also find that $B-V, U-B$ are the best BaSeL colours to derive $\log g$ and $[\mathrm{Fe} / \mathrm{H}]$ when $T_{\text {eff }}$ is fixed. Since $b-y$ gives good $T_{\text {eff }}$ estimates, this hence suggests that the $B-V, U-B, b-y$ combination, i.e. a combination of Johnson and Strömgren photometric indices, should be able to produce reliable simultaneous estimates of the 3 fundamental parameters. This suggestion is developed in the next part.

\subsection{Best synthetic colour combination}

Figures 7 and 8 show the $T_{\text {eff }}-[\mathrm{Fe} / \mathrm{H}]-\log g$ results by using the prescription suggested in the previous paragraph, namely, $B-V, U-B, b-y$. The results are also summarized in Table 2, where they are quoted as BaSeL ${ }^{c}$.

A general comparison with the other methods shows that the BaSeL solutions are very satisfactory for the 3 fundamental parameters: effective temperatures are in excellent agreement, and $[\mathrm{Fe} / \mathrm{H}]$ and gravities show good agreement. The improvement is clear when compared with the original results presented in Figs. 1 and 2 because there is no longer any systematic trend towards lower temperatures (see Table 2) and because the determinations of $\log g$ and $[\mathrm{Fe} / \mathrm{H}]$ are also much better, without systematic deviations.

There is only one exception (HD 46304): its $T_{\text {eff }}$ is in perfect agreement with its spectroscopic value, but the predicted $\log g$ and $[\mathrm{Fe} / \mathrm{H}]$ are still low. While the BaSeL contours are consistent with the Templogg gravity only at the $2-\sigma$ level, the metallicity predicted from the BaSeL models is poor in comparison to the result of the Templogg calibration $(\Delta[\mathrm{Fe} / \mathrm{H}] \simeq 0.7)$. What could explain this persistent difference? It is worth noticing that this star has a large $v \sin i\left(200 \mathrm{~km} \mathrm{~s}^{-1}\right.$, the largest in our working sample), and it is well known that high rotational velocities modify the colours. Since the expected colour effect due to rotation is typically a few hundredths of a magnitude ${ }^{2}$ in $B-V$ and increases with $v \sin i$, this is probably part of the reason why the predictions of the BaSeL models disagree with the Templogg method for this star (even if rotation is not taken into account in the Templogg method).

In conclusion, except in the case discussed before, reliable and simultaneous estimates of the 3 atmospheric parameters can be derived for F-type stars from only the three synthetic BaSeL colours, $B-V, U-B$ and $b-y$. This is a very useful criterion for further applications.

\section{Discussion}

Some of the intrinsic properties of the BaSeL library spectra can explain why synthetic Strömgren photometry does not increase the performance of $U B V$ in determining fundamental stellar parameters (most particularly, abundances and surface gravities), as follows.

The fact that the library spectra have been calibrated using broad-band photometry implies that their use in determining fundamental stellar parameters from intermediate- and narrow-band photometry cannot be expected to provide results at the same level of confidence. Our results show that, in the particular domain studied, the broad-band calibration is adapted for the $b-y$ index but not for the $m_{1}$ and $c_{1}$ indices. The reason is that, as $b-y$ has been designed to measure the continuum, its synthetic value is less affected than $m_{1}$ and $c_{1}$ by highresolution spectral features. Secondly, the library spectra have a typical resolution of $25 \AA$ in the wavelength range where the corresponding synthetic photometry is being calculated. For the Johnson broad-band $U B V$ (with passbands of halfwidths in the range 500-1000 $\AA$ ) a factor of $>2$ more flux data points are used in the numerical integrations than are used in those for synthesizing the Strömgren data (where passbands have halfwidths of order $200 \AA$ ). Since each flux point gives the integrated flux in a $25 \AA$ passband and includes the effects of spectral lines and bands, the magnitude of these effects - as well as their nonlinear variations with abundance and/or surface gravity - on the actually observed colours may be

2 These values are highly dependent of spectral type, age, and chemical composition, see for instance Maeder (1971) and Zorec (1992). 
severely distorted in the computed colours (Buser 1978). As a consequence, the accuracy of synthetic Strömgren photometry may be significantly lower than it is for $U B V$, particularly for the $m_{1}$ and $c_{1}$ indices, which are weighted sums involving three passbands each (such that errors accumulate faster than in $U B V$ indices).

Finally, a further comment on the impressively good accuracy of the BaSeL determinations for the Johnson-Strömgren $B-V, U-B, b-y$ combination (see Figs. 7 and 8 ) is in order.

As mentioned before, the Strömgren $b-y$ index provides a reliable measure of the continuum and, therefore, a good temperature index. This is particularly interesting, given the known difficulties of matching empirical and theoretical $B-V-T_{\text {eff }}$ scales to within better than about 0.03 mag (see, e.g., Sekiguchi \& Fukugita 1999) ${ }^{3}$. The fact that the $U-B$ and $B-V$ BaSeL colours give good measures of $\log g$ and $[\mathrm{Fe} / \mathrm{H}]$ is not surprising. It is well known that if one does not ask $U B V$ data to provide the temperature in the first place (e.g., by using an independent source, such as spectroscopy, spectral classification, or else suitable other photometry, such as Strömgren $b-y$ ), the sensitivities of both $U-B$ and $B-V$ can be used to full advantage for determining $\log g$ and $[\mathrm{Fe} / \mathrm{H}]$. Moreover, although these sensitivities change with temperature, they are near or even at their maxima in the F-dwarf star domain (e.g., Buser \& Kurucz 1992). This means that the derived values of $[\mathrm{Fe} / \mathrm{H}]$ and $\log g$ presented in Figs. 7 and 8 are as reliable as they can possibly be, given the uncertainties in the colours.

\section{Conclusion}

Several methods of determination of the fundamental stellar parameters $T_{\text {eff }}, \log g$ and [Fe/H] are compared for nine single F stars. Particular attention has been paid to the simultaneous predictions of the BaSeL models in two photometric systems, Johnson and Strömgren. We show that using all photometric data is not the best strategy to obtain reliable simultaneous determinations of $T_{\mathrm{eff}}, \log g$ and $[\mathrm{Fe} / \mathrm{H}]$ with the BaSeL models in the temperature range relevant for this paper, because of intrinsic limitations of the BaSeL library. As a matter of fact, if one uses all five available photometric indices $\left(B-V, U-B, b-y, m_{1}\right.$ and $c_{1}$ ), only the agreement with spectroscopic determination for the effective temperature is good, although the BaSeL-derived temperatures are slightly but systematically lower. The discrepancies in gravity and metallicity are however rather high, with the BaSeL predictions being too low. Alternatively, we show that the best results are obtained by using $B-V, U-B, b-y$ in combination. This BaSeL combination is the best because on the one hand the $b-y$ synthetic index gives reliable and accurate estimates of the effective temperature and, on the other

\footnotetext{
3 The $b-y$ index is less vulnerable than $B-V$ to the secondary effects of surface gravity and metallicity, even at the low spectral resolution given by the model spectra.
}

hand, $B-V$ and $U-B$ give good estimates of $[\mathrm{Fe} / \mathrm{H}]$ and the surface gravity. We wish to emphasize that, if Strömgren photometry is shown here to be less performing than $U B V$ as a device for determining fundamental stellar parameters (and, most particularly, abundances and surface gravities), this is due to the fact that the BaSeL library spectra have been calibrated by colour-calibration in $U B V R I J H K L M$; it is not, of course, due to an intrinsic superiority of the $U B V$ system over the Strömgren system. Thus, because the BaSeL library has only been calibrated in the UBVRIJHKLM colours, the best parameters derivations come from this system. Whilst parameters derivations in equivalent bandwidth systems such as Washington seem to be as good with the BaSeL libraries than with empirical methods (Lejeune 1997), parameter derivations from the BaSeL library in narrow band systems such as Strömgren photometry seem to be of poorer quality.

This work thus strongly suggests that the BaSeL library should be calibrated in various photometrical systems, in various bandwidth (maybe using higher resolution synthetic stellar spectra libraries) in order to represent in the best way the variety of the knowledge of stellar spectral energy distributions that exists today in various photometric systems.

We found that the best combination to determine stellar parameters within the BaSeL library, even with its limitation, was to use a combination of two Johnson colours, $U-B$ and $B-V$, and a Strömgren colour, $b-y$.

We also note that the agreement between Templogg and BaSeL for the hottest stars of the sample could be especially useful in view of the well known difficulty of spectroscopic determinations for fast rotating stars.

As far as the astrophysical applications are concerned, the BaSeL synthetic colours are of particular interest in evolutionary synthesis and colour-magnitude diagram studies of stellar populations, such as open clusters and young associations, where (i) F-type stars are highly common and, of course, (ii) a vast abundance of data are available in $B-V, U-B$ and $b-y$ colours. Concerning the determination of the atmospheric parameters of the COROT potential targets, a result of the present analysis is that all the methods give consistent solutions. In the context of the further $\sim 1000$ potential targets of the COROT exploratory programme, it will be interesting to compare the results of the BaSeL models with those of automated spectral analysis methods (e.g. Katz et al. 1998; Bailer-Jones 2000).

Acknowledgements. We would like to thank J.-L. Vergely for providing extinction calculations as well as J. C. Bouret, C. Catala and D. Katz for their participation in the spectral characterization of the stars. We are fully indebted to R. Kurucz for all the codes and theoretical data so generously displayed. E. L. and F. L. are supported by PPARC postdoctoral fellowship. Th. Lejeune gratefully acknowledges financial support from the Swiss NSF (grant 20-53660.98 to R.B.), and Th. Lüftinger from the "Fonds zur Förderung der wissenschaftlichen Forschung". F. C. thanks FAPERJ for partial 
funding, through the grant E-26/171.368/1999. This research has made use of the QMW Starlink resource facilities and the SIMBAD database operated at CDS, Strasbourg, France.

\section{References}

Baglin, A., et al. 1998, IAU Symposium, 185, New Eyes to See Inside the Sun and Stars, ed. F.-L. Deubner, J. Christensen-Dalsgaard, \& D. Kurtz, 301

Bailer-Jones, C. A. L. 2000, A\&A, 357, 197 [astro-ph/0003071]

Bruzual, G., Barbuy, B., Ortolani, S., et al. 1997, AJ, 114, 1531

Buser, R. 1978, A\&A, 62, 411

Buser, R., \& Kurucz, R. L. 1992, A\&A, 264, 557

Carlberg, R. G., Dawson, P. C., Hsu, T., \& Vandenberg, D. A. 1985, ApJ, 294, 674

Catala, C., Mangeney, A., Gautier, D., et al. 1995, Astronomical Society of the Pacific Conf. Ser., 76, 426

Cayrel de Strobel, G., Soubiran, C., Friel, E. D., Ralite, N., \& Francois, P. 1997, A\&AS, 124, 299

Cuisinier, F., Buser, R., Acker, A., et al. 1994, A\&A, 285, 943

Cuisinier, F., Lejeune, T., \& Buser, R. 1996, IAU Symp. 171, ed. R. Bender, \& R. L. Davies, 355

Donati, J.-F., Semel, M., Carter, B. D., Rees, D. E., \& Cameron, A. C. 1997, MNRAS, 291, 658

ESA 1997, The Hipparcos and Tycho Catalogues, ESA-SP 1200

Katz, D., Soubiran, C., Cayrel, R., Adda, M., \& Cautain, R. 1998, A\&A, 338, 151

Künzli, M., North, P., Kurucz, R. L., \& Nicolet, B. 1997, A\&AS, 122, 51

Kupka, F., Piskunov, N., Ryabchikova, T. A., Stempels, H. C., \& Weiss, W. W. 1999, A\&AS, 138, 119

Kurth, O. M., Fritze-v. Alvensleben, U., \& Fricke, K. J. 1999 , A\&AS, 138, 19

Kurucz, R. L. 1993, CD-ROM, 13, 14

Lastennet, E., Lejeune, T., \& Valls-Gabaud, D. 1996, ASP Conf. Ser., 90, 157

Lastennet, E., Lejeune, T., Westera, P., \& Buser, R. 1999a, A\&A, 341, 857
Lastennet, E., Valls-Gabaud, D., Lejeune, T., \& Oblak, E. 1999b, A\&A, 349, 485

Lejeune, T. 1997, Ph.D. Thesis, Observatoire Astronomique de Strasbourg, France

Lejeune, T., Cuisinier, F., \& Buser, R. 1997, A\&AS, 125, 229

Lejeune, T., Cuisinier, F., \& Buser, R. 1998, A\&AS, 130, 65

Lejeune, T., Westera, P., \& Buser, R. 2000, in preparation

Lignières, F., Catala, C., Katz, D., et al. 1999, Joint European and National Astronomical Meeting (JENAM 99), 7-11 Sept. 1999, Toulouse (France)

Maeder, A. 1971, A\&A, 10, 354

Marsakov, V. A., \& Shevelev, Y. G. 1995, Bull. Inform. CDS, 47,13 [MS95]

Michel, E., Baglin, A., et al. 1998, Structure and Dynamics of the Interior of the Sun and Sun-like Stars, SOHO 6/GONG 98 Workshop Abstract, June 1-4, 1998, Boston, Massachusetts

Moon, T. T. 1985, Com. Univ. London Obs., 78, 1

Moon, T. T., \& Dworetsky, M. M. 1985, MNRAS, 217, 305

Napiwotzki, R, Schönberner, D., \& Wenske, V. 1993, A\&A, 268,653

North, P., Künzli, M., \& Nicolet, B. 1994, 22nd GA of the IAU, the Hague

Perryman, M. A. C., et al. 1997, A\&A, 323, L49

Piskunov, N. E. 1992, in Stellar magnetism, ed. Y. V. Glagolevskij, \& I. I. Romanyuk, Nauka, St. Petersburg, 92

Sekiguchi, M., \& Fukugita, M. 1999 [astro-ph/9904299]

van't Veer-Menneret, C., \& Mégessier 1996, A\&A, 309, 879

Vergely, J.-L. 1998, Ph.D. Thesis, Observatoire Astronomique de Strasbourg, France

Weiss, A., \& Salaris, M. 1999, A\&A, 346, 897

Welsh, B. Y., Vedder, P. W., Vallerga, J. V., \& Craig, N. 1991, ApJ, 381, 462

Westera, P., Lejeune, T., \& Buser, R. 1999, ASP Conf. Series 192, ed. I. Hubeny, S. R. Heap, \& R. H. Cornett, 203 [astro-ph/9906064]

Zakhozhaj, V. A., \& Shaparenko, E. F. 1996, Kinematika Fiz., Nebesn. Tel., 12, part No. 2, 20-29, [ZS96]

Zorec, J. 1992, Hipparcos, Goutelas 1992, ed. D. Benest, \& C. Froeschlé, 407 